\title{
THE EFFECT OF QUININE ON RABIES IN DOGS.*
}

\author{
Virgil H. MoOn.
}

(From the Memorial Insticute for Infectimus Diserses, Chicago.)

The preventive treatment of rabies as devised by Pasteur is efficient only during the interval preceding the development of active symptoms, and as is well known, this treatment offers no hope after those symptoms become manifest. There is sufficient reason then why efforts should be made to find a curative remedy for rabies. It is conceded that rabies is an infectious disease, and many investigators go a step farther and class it among the diseases produced by protozoan parasites. The successful treatment of other diseases of parasitic nature, notably malaria, sleeping sickness, syphilis, etc., by internal medication should encourage efforts to discover an agent which will be effective against rabies. The fact that the preventive treatment now in use depends on the patient's system to build up its own protective substances or immunity, lends encouragement in this way-the remedy sought need not be one of sufficient strength to kill the infecting organisms outright. If a substance were found which would merely inhibit or retard the development of the infection, there is reason to believe that the system might respond by the development of antibodies as it does when injected with the attenuated virus in the course of preventive treatment. It was in pursuance of this idea that the following experiments were made.

Dogs were inoculated with rabid brain material and allowed to develop active symptoms of rabies. The technic of inoculation was as follows: a portion of the hippocampus or cerebral cortex of a rabid animal was rubbed into an cmulsion with normal salt solution. With a small needle a few drops of this emulsion were then injected into and around the ulnar nerve at the elbow or a long, slender needle was inserted in the lower conjunctival sack below the eyeball and extended back until the point entered or passed through the optic foramen. The injection was thus made

* Received for publication June a, tol 3 . 
directly into the optic tract, or subdurally. The latter method of course necessitated general anesthesia, but the results were more uniform than by the other method, and the incubation period shorter. When active symptoms of rabies developed, as unusual excitability, restlessness. changed quality of the bark. decreased appetite. sometimes difficulty of swallowing, muscular spasms. paralysis of the legs, etc.. the dog was given very large doses of quinine sulfate several times daily. while another dog inoculated at the same time and under the same conditions was allowed to go untreated as a control. The quinine was administered in capsules around which was stitched a thin covering of fresh tough meat as from the flank of beef. The dog would usually bolt these without chewing. but if he became unable to swallow, as happened several times, the same quantity of quinine bi-sulfate was given in solution hypodermically. The daily amount given a 6 or 7 kilogram dog was from I. 0 to $\mathrm{I} .6 \mathrm{gm}$.. usually in three doses. This is equivalent to from 12 to $18 \mathrm{gms}$. daily for an average man, in proportion to the body weight. The medication was thus pushed to the limit to secure the full physiological, bordering on toxic, effect. The idea was that in order to test thoroughly the effect of quinine. the system should be kept saturated with it. No ill effects followed. and the results seem encouraging as shown by the following data.

\section{EXPERIMENT I.}

May I4. I9I 2. Dogs I and 2 were inoculated in the optic tract with emulsion of rabid brain.

May 25. Both dogs excitable and vicious, snarling and biting when disturbed.

May 26. Symptoms continued. Quinine treatment begun on Dog 2.

June 3. Dog I (untreated) was vicious and savage. bark high pitched and prolonged, was getting thin. attacked food savagely. ate with difficulty, was violent and bruised the head and face against the cage.

Dog 2 was weak in the hind legs, partially paralyzed. bark hoarse and prolonged. 
June 7. Dog I dead. Examination of the brain showed Negri bodies, large and numerous.

Dog 2 seemed nearer normal, the bark was still unnatural but the dog was not savage, nor disposed to bite.

June 9. Dog 2 apparently normal.

June II. Quinine treatment discontinued, a total of $14.6 \mathrm{gms}$. having been given.

For a month following the treatment the dog seemed sullen and irritable of temper and would bark, growl and snap if approached. Guinea-pig inoculations to determine if the saliva were infective gave negative results.

August I. At this time the dog's disposition seemed normal. He was perfectly docile, wagged the tail eagerly when approached and gratefully accepted petting.

August 26. Two and one-half months after the close of the treatment the dog died of unknown cause. I was unable to make an autopsy to determine the cause of death, as I was in the hospital as a typhoid patient at the time. Examination of the brain by Dr. Weber of the Chicago Health Department showed no Negri bodies.

\section{EXPERIMENT 2.}

June 6, rgr 2. Dogs 3 and 4 inoculated in the optic tract with emulsified rabid brain.

July ro. Dog 3 was very excitable and the bark high pitched. He continued to show signs of rabies, but the disease was allowed to run its course as a control to Dog 4. On July 23, Dog 3 died after a characteristic course. Examination of the brain showed Negri bodies.

Dog 4 showed no definite symptoms until August 3, almost six weeks after inoculation. The dog became excitable; the barks were not distinct but run together and of higher pitch than normally. Quinine treatment was begun.

August 5. Dog 4 was inattentive and disposed to lie down. was unsteady on his feet and partially paralyzed in the hind legs. Had difficulty in eating, and was unable to swallow the capsule of quinine wrapped in meat; quinine given by injection. 
August 6. The dog's condition was considerably improved; he was able to eat and take the capsules as before.

August ro. Dog apparently normal.

August 12. Quinine discontinued, a total of 13 gms. having been given.

June 5,1913 . Dog 4 is alive, healthy and vigorous, apparently a normal animal.

\section{EXPERIMENT 3 .}

January 14, I9I3. Dogs 5 and 6 were inoculated with emulsion of rabid brain.

February 13. Dog 5 showed sudden and extremely severe onset of symptoms. Incoördination and partial paralysis first noticed in the morning. Severe tonic spasms and inability to swallow. The spasms increased in severity and length, and he died apparently of spasm of the respiratory muscles in the evening of the same day. Brain examination showed Negri bodies.

February I5. Dog 6 restless and excited. whined and barked continuously and bruised the face by striking against the cage. Quinine treatment begun at once. A guinea-pig was inoculated with saliva taken from the dog's mouth. The pig died Io days later with typical signs of rabies and examination of the brain showed numerous Negri bodies.

February i8. The dog had marked muscular spasms, was very excitable, and unable to swallow. Quinine given hypodermically.

February 20. The dog showed partial paralysis of the legs. muscular spasms continued and the general condition was such that there seemed no hope of the dog living. The only favorable sign was that he was again able to swallow.

February 21. The general condition showed some improvement and the spasms were not so severe.

During the following days the condition improved steadily.

February 24. The dog apparently normal. Treatment discontinued, $7.8 \mathrm{gms}$. of quinine having been given.

June 5, I9I3. Dog 6 is a playful, healthy, normal animal. 
SUMMARY.

I have endeavored to maintain a conservative view of the results. However the following points stand out:

Of the three dogs treated two are alive and healthy at the present writing and one died of obscure cause two and one half months after treatment.

The control animals in every case died with the characteristic symptoms and pathological changes of rabies.

It is admitted by some authorities that dogs occasionally recover spontaneously from rabies. If we assume then that this was the case, and that the quinine had nothing to do with the results. we are confronted by an alternative of extreme improbability. One of the treated animals may have recovered spontaneously; that this should occur a second time is extremely improbable; that it should happen three times consecutively, and that the untreated control should die in every case is little short of impossible. However the above cases are not taken to mean that an absolute cure for developed rabies has been found. It is known that in rabies as in other diseases there are variations in severity and virulence in different cases. It is possible that these cases were of sufficiently moderate severity that the quinine furnished barely sufficient aid to enable the system to throw off the disease, and that the same result would not have followed in very severe cases. I do not assume that because quinine has been effective in dogs that it will be equally so in human cases, though this is not improbable. Viewed most conservatively these results are encouraging and indicate that the medical treatment of developed hydrophobia should not be regarded as hopeless. Should quinine not prove efficient when put to more severe tests, other agents should be given thorough trial experimentally. Upon the experimental basis already provided one would be justified in applying the same method, or rather one would not be justified in withholding the same method of treatment, from a patient suffering from hydrophobia.

In the instances above described quinine treatment was begun on the day that definite symptoms were first evident, and in human cases the quinine should be used as early as possible and freely 
enough to get the full effect. The use of sedatives to quiet nervousness and excitability is in order; to prevent pharyngeal spasms on attempting to swallow, spraying the pharynx with cocaine solution has been resorted to. Curare has been used hypodermically in minute doses to relieve extreme muscular spasms in hydrophobia. Obviously all means possible should be employed to conserve the patient's strength, as exhaustion weakens the resistance to any disease. These measures are not offered as a substitute for Pasteur's preventive treatment of hydrophobia; no other treatment than that is to be considered during the incubation stage of the disease. But when active symptoms have developed, the Pasteur treatment is hopeless. while the treatment just described may offer some hope if instituted without delay. 